\title{
The development of hospital-based palliative care services in public hospitals in the Western Cape, South Africa
}

\author{
L Gwyther, ${ }^{1}$ MB ChB, FCFP, MSc Pall Med; R Krause, ${ }^{2}$ MB ChB, MFamMed, MPhil Pall Med; C Cupido, ${ }^{3}$ MB ChB, FCP (SA); \\ J Stanford, ${ }^{4} \mathrm{MB} \mathrm{ChB}$, MPhil Pall Med; H Grey, ${ }^{4}$ BSc Nursing, Dipl Oncol Nursing, MBA, PGD Pall Med; T Credé, ${ }^{5}$ MB ChB, FCP (SA); \\ A de Vos, ${ }^{6}$ MSocSci Clinical Social Work; J Arendse, ${ }^{7}$ BTech Oncology, Dipl Midwifery, Palliative Care Cert; P Raubenheimer, ${ }^{8}$ MB BCh, FCP (SA) \\ ${ }^{1}$ Palliative Medicine, Division of Family Medicine, Faculty of Health Sciences, University of Cape Town, and Hospice Palliative Care Association of \\ South Africa, Cape Town, South Africa \\ ${ }^{2}$ Palliative Medicine, Division of Family Medicine, Faculty of Health Sciences, University of Cape Town, and Groote Schuur Hospital, Cape Town, \\ South Africa \\ ${ }^{3}$ Division of General Medicine, Faculty of Health Sciences, University of Cape Town, and Victoria Hospital, Cape Town, South Africa \\ ${ }^{4}$ Knysna-Sedgefield Hospice, Knysna, South Africa \\ ${ }^{5}$ Division of General Medicine, Faculty of Health Sciences, University of Cape Town, and Mitchell's Plain Hospital, Cape Town, South Africa \\ ${ }^{6}$ Social Work Services, Groote Schuur Hospital, Cape Town, South Africa \\ Groote Schuur Hospital, Cape Town, South Africa \\ ${ }^{8}$ Division of General Medicine, Department of Medicine, Faculty of Health Sciences, University of Cape Town, and Groote Schuur Hospital, \\ Cape Town, South Africa
}

Corresponding author: L Gwyther (liz.gwyther@uct.ac.za)

With the recent approval of a South African (SA) National Policy Framework and Strategy for Palliative Care by the National Health Council, it is pertinent to reflect on initiatives to develop palliative care services in public hospitals. This article reviews the development of hospital-based palliative care services in the Western Cape, SA. Palliative care services in SA started in the non-governmental sector in the 1980s. The first SA hospital-based palliative care team was established in Charlotte Maxeke Johannesburg Academic Hospital in 2001. The awareness of the benefit of palliative care in the hospital setting led to the development of isolated pockets of excellence providing palliative care in the public health sector in SA. This article describes models for palliative care at tertiary, provincial and district hospital level, which could inform development of hospital-based palliative care as the national policy for palliative care is implemented in SA.

S Afr Med J 2018;108(2):86-89. DOI:10.7196/SAMJ.2018.v108i2.12524

With the recent approval of a South African (SA) National Policy Framework and Strategy for Palliative Care by the National Health Council, it is pertinent to reflect on initiatives to develop palliative care services in public hospitals. This article reviews the development of hospital-based palliative care services in the Western Cape, SA.

Internationally, palliative care has been integrated into hospital services, with Higginson ${ }^{[1]}$ describing hospital palliative care teams in the UK palliative care needs assessment report in 1997. Weissman and Meier ${ }^{[2]}$ describe the importance of an indicator guide to identify patients in need of palliative care in the hospital setting in the USA. They also stress the need to improve palliative care skills in clinicians caring for patients with serious illnesses and indicate specific criteria for referral to a hospital palliative care service.

The importance of integration of palliative care into health services was highlighted with the adoption of the 2014 World Health Assembly (WHA) resolution, ${ }^{[3]}$ 'Strengthening of palliative care as a component of comprehensive care throughout the life course'. The WHA resolution draws on the World Health Organization's definition of palliative care ${ }^{[4]}$ as 'an approach that improves the quality of life of patients and their families facing problems associated with lifethreatening illness, through the prevention and relief of suffering, the early identification and impeccable assessment and treatment of pain and other problems, physical, psychosocial and spiritual'.

The resolution states that palliative care is an 'ethical responsibility of health systems, and that it is the ethical duty of health care professionals' to relieve suffering. Palliative care is applicable to any diagnosis that may result in a person's death. It is applicable early in the diagnosis of a life-threatening illness, in conjunction with treatment that is aimed at containing the illness. Therefore, all healthcare professionals should be competent to provide palliative care alongside disease-specific treatment. Referral for specialist palliative care, such as may be provided by a hospice, would then only be for patients with challenging symptoms and/or psychosocial issues.

\section{Development of palliative care in South Africa}

Palliative care services in SA started in the non-governmental sector in the 1980s. Hospices were established in communities that could afford to support them and initially provided end-of-life care, mainly to cancer and HIV patients - from the mid-1990s onwards. Depending on admission criteria to a hospice service, patients with cancer, HIV, end-stage organ failure, progressive neurological disorders and tuberculosis (TB), particularly drug-resistant TB, receive palliative care - usually in the patient's home. Hospice services in SA are nurse led with support from an interdisciplinary team, including social workers, spiritual counsellors and doctors. South Coast Hospice in Port Shepstone, KwaZulu-Natal initiated an integrated community-based home care service in response to the increasing number of HIV patients requiring palliative care. ${ }^{[5]}$ This integrated approach developed partnerships with the government 
sector, leading to a greater awareness of the benefits of palliative care by the state sector. The first hospital-based palliative care team in SA was established in Charlotte Maxeke Johannesburg Academic Hospital in 2001. ${ }^{[6]}$ The awareness of the benefits of palliative care in the hospital setting led to the development of isolated pockets of excellence providing palliative care in the public health sector in SA. Chief of these services is Wits Palliative Care, Gauteng Centre of Excellence for Palliative Care, Chris Hani Baragwanath Hospital in Soweto. The programme developed from a hospital-based palliative care team established in 1999, which ran the N'Doro project funded by Irish Aid for 3 years (2003 - 2006), providing specialist palliative care services, outreach visits to the Soweto community, consultations for patients in Chris Hani Baragwanath Hospital, as well as training of healthcare professionals, conducting research and undertaking advocacy activities for palliative care ${ }^{[2]} \mathrm{A}$ research study undertaken by the Health Systems Research Unit at the South African Medical Research Council, evaluating the N'Doro project, demonstrated a reduction in hospital costs and additional benefits to the hospital care system by reducing congestion and ensuring that hospital beds are freed for acute care. ${ }^{[7]}$

\section{Palliative care in Western Cape hospitals}

In the Western Cape, palliative care services extended into public service hospitals, with various models being introduced and developed based on local needs. The development of these hospital services has been underpinned by research that demonstrated the benefits of palliative care in hospitals. Drawing on the UK Gold Standards Framework (GSF), Drs L van Niekerk and C Cupido established a palliative care service - the Abundant Life programme at Victoria Hospital, a large district hospital (with specialist services) in Cape Town. Seed funding for nursing and administrative support was provided by donors, especially Rotary ${ }^{[8]}$ Patients, initially mainly those with advanced organ failure, and family members attend an outpatient group clinic held fortnightly. A multidisciplinary approach assists in educating the patient and family about their illness and prognosis, providing practical advice on coping with the illness, as well as emotional support. The Abundant Life patients were shown to have fewer admissions, fewer days in hospital, higher rates of supported home deaths and lower hospital costs than the control group ${ }^{[9]}$ The referrals have grown from 200 patients in the first 2 years and 70 active patients at the end of 2011, to >600 referrals for 2016 and 250 active patients. The service has extended from the medical wards into the surgical wards, referral criteria have been broadened and a part-time palliative care nurse has been appointed to a provincial position at Victoria Hospital. Donor funding continues to support a large part of this programme.

Groote Schuur Hospital (GSH), a large tertiary teaching hospital in Cape Town, introduced palliative care in 2011, when Dr T Credé, head of the medical Emergency Unit at the hospital, identified the need to improve end-of-life care for patients presenting with acute or chronic conditions, who were not expected to survive beyond 24 hours and were often not prioritised for admission, given the pressure with regard to acute and elective beds. Four unfunded palliative care beds were opened in a ward with bed space to ensure patients admitted from the Emergency Unit and their families received the appropriate care. The ward staff, St Luke's hospice and the Hospice Palliative Care Association of SA provided training and mentorship to GSH staff, creating a space for dignified endof-life care in the hospital. Having dedicated beds where patients are cared for in the last 24 hours of their life, has not only led to a new expertise in the treatment of such patients, but has also enabled new nurse-led expertise in the hospital. Nurses have generally been very positive about the experience of re-focusing comprehensive care with regard to this cohort of challenging patients. The main cohort of patients seen in the Emergency Unit are those with major strokes, and the median length of stay is 25 hours. The success of this project, together with the research findings from StuartClark et al., ${ }^{[9]}$ which demonstrated a $42 \% 12$-month mortality rate among medical patients at GSH, and research of Van Niekerk and Raubenheimer ${ }^{[10]}$ who identified a high proportion of patients expected to benefit from end-of-life and palliative care, were used to motivate for a permanent hospital palliative care team for medical patients with acute conditions admitted to GSH. Van Niekerk and Raubenheimer ${ }^{[10]}$ reported that $20.3 \%$ of patients in general medical wards had life-limiting illnesses, potentially requiring palliative care. They also commented on the young age of patients, the high prevalence of end-stage renal failure and HIV/TB among those assessed as needing palliative care. The GSH palliative care service is run by a nurse-led, doctor-supported team introduced initially into the acute medical wards, but subsequently expanded to the Emergency Unit, the Renal Unit (particularly for patients with end-stage renal disease not accepted into the provincial renal replacement programme) and more recently in 2017 to the surgery and gynaecology wards. The hospital palliative care team consists of 2 palliative care professional nurses, 2 auxiliary social workers, spiritual care volunteers and medical support from the University of Cape Town palliative care team in the Division of Family Medicine. There is a very close working relationship with the departments of Social Work, Allied Health and Nursing in the hospital. The main objectives of the team are to provide a comprehensive palliative care assessment, assist with discharge planning using the multidisciplinary team, optimise pain and symptom control, provide psychosocial and spiritual support to the patient and their families, liaise with and refer to community-based services and follow up telephonically, including bereavement calls. The team develops an individual care plan for each patient, which guides care once the patient is discharged home. The discharge plan includes referral to a community-based service, often the hospice, which enables the patient to live as actively as possible with their illness and supports their family in providing care at home. From January to December 2016 (in the medical pavilion only), the GSH palliative care team received 79 referrals per month, provided care to 852 unique patients and gave 861 telephonic consultations to discharged patients. The most common conditions for which referrals are received are end-stage cardiac failure (44\%), metastatic cancer (40\%), end-stage renal failure - not for the renal replacement programme (19\%), progressive neurological conditions and endstage chronic obstructive pulmonary disease (COPD) ( $8 \%$ each).

\section{Hospital/hospice partnerships}

District hospitals have also introduced palliative care services, mainly in conjunction with local hospice support. Stellenbosch Hospital has provided a ward to Stellenbosch Hospice and provides medicines and support services to the 10-bed hospice. Knysna Provincial Hospital staff meet fortnightly with the staff from the Knysna-Sedgefield and Plettenberg Bay hospices to discuss challenging problems. Patients are admitted to the palliative care programme if they have been admitted to the hospital at least twice in 4 weeks or have been assessed as terminally ill and are currently in hospital. Patients are seen by the palliative care team in the medical wards at the hospital and a palliative care plan is developed for each patient referred. In addition, Knysna-Sedgefield Hospice staffs the 6-bed intermediate care facility 
Table 1. Identifying patients who require palliative care: Summary of the Supportive and Palliative Care Indicators Tool ${ }^{[13]}$

General indicators for palliative care referral

Performance status is poor or deteriorating (the person is in bed or a chair for $\geq 50 \%$ of the day); reversibility is limited

Dependent on others for most care needs owing to physical and/or mental health problems

$\geq 2$ unplanned hospital admissions in the past 6 months

Significant weight loss (5 - 10\%) over the past 3 - 6 months, and/or a low body mass index

Persistent troublesome symptoms, despite optimal treatment of underlying condition(s)

Patient asks for supportive and palliative care, or treatment withdrawal

Clinical indicators for $\geq 1$ advanced conditions

E.g. cancer, heart/vascular disease, kidney disease, respiratory disease, liver disease, dementia/frailty, neurological disease

Indicators for infectious diseases are still to be agreed with regard to the South African setting

on the hospital grounds and occasionally patients requiring palliative care are referred to this facility. Patients are discussed by the team and a palliative care plan is developed for each patient to ensure a holistic approach to symptom management. This comprehensive integrated approach is feasible in small communities, but has the same barriers to access to palliative care as larger centres.

\section{Identifying patients who require palliative care}

The main barrier to palliative care access is the identification of palliative care patients. The GSF Prognostic Indicator Guidance $(\mathrm{PIG})^{[1,12]}$ and the Supportive and Palliative Care Indicators Tool (SPICT $)^{[13,14]}$ are documents that are used internationally to assist the clinician to implement palliative care principles of pain and symptom management with psychosocial and spiritual support. At GSH and Victoria Hospital a simplified and modified 1-page tick-sheet based on the GSF-PIG is used to assist with the identification of palliative care patients (including HIV patients). There is a need to develop and validate a SA-specific referral tool for palliative care in hospital and community settings. Referrals are 'open' to all health professionals, and can be from doctors, nurses, allied health professionals and social workers involved in the care of ward patients (Table 1).

Hand-in-hand with a simplified referral form, basic education and awareness regarding palliative care among all healthcare professionals contribute to a steady referral pattern. Seventy-two nurses and social workers from GSH underwent the 40-hour training programme in palliative care presented by the Hospice Palliative Care Association of SA to ensure that they are able to assist the hospital palliative care team and identify patients who would benefit from such care. Therefore, with the minimal resources allocated and low levels of funding, the GSH palliative care team have been able to prioritise a generalist and inclusive approach to palliative care, with the specialist sister acting not only as an expert clinician, but also as a mentor and trainer of ward staff and the allied health support team. The ward staff and associated allied health teams have been given the training and support they need to provide palliative care.

\section{Linking with community resources}

To ensure comprehensive continued care of patients, hospital-based palliative care services have understood the fundamental need to develop strong ties with community resources. The palliative care team approach has been instrumental in establishing a strong referral process with post-discharge primary home-based care, either to hospices or provincial non-governmental organisation (NGO)-contracted homebased carers. A combined care approach ensures easy referral, care plans that are patient centred and better team support.

Palliative care as a hospital-based specialty is still in its infancy in SA and there is enormous opportunity for growth in a traditional specialty-based setting, such as a hospital. The number of hospitalised patients who are in their last year of life, presents a unique opportunity for identifying patients and instituting care plans that are patient centred and can also be followed up in the community setting. Capturing data on hospital palliative care is essential and provides important opportunities for research and advocacy around palliative care and for updating African palliative care guidelines for life-threatening illnesses. A recent needs assessment of palliative care in SA indicated that only $17.8 \%$ of patients who could benefit from palliative care at the end of their lives receive such care. ${ }^{[13]}$ The NGO sector, traditional provider of palliative care, cannot meet the total need for palliative care in SA; the government should also not expect NGOs to substitute for governmental obligations. ${ }^{[14]}$ It is therefore essential that all healthcare professionals in SA are able to identify people needing palliative care, initiate basic palliative care to ensure better care in the last year of a patient's life and link such patients with community services to continue provision of palliative care in the patient's home.

This article describes models for palliative care at tertiary, provincial and district hospital level, which could inform the development of hospital-based palliative care as the national policy for palliative care is implemented in SA. Palliative care is an essential component of universal health coverage. It 'is recognised as fundamental to improving quality of life, well-being, comfort and human dignity for individuals. The integration of palliative care into hospitals recognises these imperatives and contributes to better quality of care with a strong, patient-centred approach for people with lifethreatening illnesses and their families.

Acknowledgements. We acknowledge the staff of palliative care hospital teams, as well as the patients and their families.

Author contributions. RK, LG and PR conceived the article. LG wrote the introduction and development sections. RK, PR and TC composed the section on GSH. LG, HG and JS were responsible for the partnership section. RK, LG, PR and CC wrote on identifying patients. LG and RK compiled the community resources section. All co-authors reviewed the article, added comments and made editorial changes, which were included by LG.

Funding. None.

Conflicts of interest. None.

1. Higginson IJ. Health care needs assessment: Palliative and terminal care. In: Stevens A, Raftery J, eds. Health Care Needs Assessment. Oxford: Wessex Institute of Public Health Medicine, 1997:183-260. 2. Weissman DE, Meier DE. Identifying patients in need of a palliative care assessment in the hospital setting: A consensus report from the center to advance palliative care. J Palliat Med 2011;14(1):17-23. https://doi.org/10.1089/jpm.2010.0347

3. World Health Assembly. Strengthening of palliative care as a component of comprehensive care throughout the life course. http://apps.who.int/gb/ebwha/pdf_files/WHA67/A67_R19-en.pdf (accessed 8 December 2017).

4. World Health Organization. Definition of palliative care. http://www.who.int/cancer/palliative definition/en/ (accessed 8 December 2017). 
5. Defilippi K. Integrated community-based home care: Striving towards balancing quality with coverage in South Africa. Ind J Palliat Care 2005;11(1):34. https:// doi.org/10.4103/0973-1075.16643

6. Kirk J, Collins K. Difference in quality of life of referred hospital patients after hospital palliative care team intervention: Clinical practice. S Afr Med J 2006;96(2):101-102.

7. Hongoro C, Dinat N. A cost analysis of a hospital-based palliative care outreach program: Implications for expanding public sector palliative care in South Africa. J Pain Symptom Manage 2011;41(6):1015 1024. https://doi.org/10.1016/j.jpainsymman.2010.08.014

8. DesRosiers T, Cupido C, Pitout E, et al. A hospital-based palliative care service for patients wit advanced organ failure in sub-Saharan Africa reduces admissions and increases home death rates. J Pain Symptom Manage 2014;47(4):786-792. https://doi.org/10.1016/j.jpainsymman.2013.05.021

9. Stuart-Clark H, Vorajee N, Zuma S, et al. Twelve-month outcomes of patients admitted to the acute general medical service at Groote Schuur Hospital. S Afr Med J 2012;102(6):549-553. https://doi org/10.7196/samj. 5615

10. Van Niekerk L, Raubenheimer PJ. A point-prevalence survey of public hospital inpatients with palliative care needs in Cape Town, South Africa. S Afr Med J 2013;104(2):138-141. https://doi. palliative care needs in

11. Gold Standards Framework. GSF prognostic indicator guidance. http://www.goldstandardsframework org.uk/cd-content/uploads/files/General\%20Files/Prognostic\%20Indicator\%20Guidance\%20 October\%202011.pdf (accessed 29 March 2017)
12. O'Callaghan A, Laking G, Frey R, Robinson J, Gott M. Can we predict which hospitalised patients are in their last year of life? A prospective cross-sectional study of the Gold Standards Framework Prognostic
Indicator Guidance as a screening tool in the acute hospital setting. Palliat Med 2014;28(8):1046-1052. https://doi.org/10.1177/0269216314536089

13. Supportive and Palliative Care Indicators Tool. http://www.spict.org.uk/the-spict/ (accessed 29 March 2017).

14. Highet G, Crawford D, Murray SA, Boyd K. Development and evaluation of the Supportive and Palliative Care Indicators Tool (SPICT): A mixed-methods study. BMJ Support Palliat Care 2014;4(3):285-290. https://doi.org/10.1136/bmjspcare-2013-00048

15. Gwyther L. How is palliative care part of the right to health? The South African evidence. PhD thesis, University of Cape Town (in progress).

16. London L. What is a human rights-based approach to health and does it matter? Health Human Rights 2008;10(1):65-80. https://doi.org/10.2307/20460088

Accepted 29 August 2017. 